\title{
Период капельного квазибесселева пучка, генерируемого аксиконом со скругленной вершиной
}

\author{
(C) В.Ю. Мыльников ${ }^{1}$, Д.В. Чистяков ${ }^{1}$, С.Х. Абдулразак ${ }^{1}$, Н.Г. Дерягин ${ }^{1}$, Ю.М. Задиранов ${ }^{1}$, \\ С.Н. Лосев ${ }^{1}$, В.В. Дюделев ${ }^{1,2}$, Г.С. Соколовский ${ }^{1}$ \\ ${ }^{1}$ Физико-технический институт им. А.Ф. Иоффре РАН, \\ Санкт-Петербург, Россия \\ ${ }^{2}$ Санкт-Петербургский государственный электротехнический университет „ЛЭТИ“, \\ Санкт-Петербург, Россия \\ E-mail: vm@mail.ioffe.ru
}

Поступило в Редакцию 30 июня 2021 г.

В окончательной редакции 13 июля 2021 r.

Принято к публикации 13 июля 2021 г.

На основе экспериментального исследования продольного распределения интенсивности квазибесселева пучка с капельной структурой центрального луча, сформированного при помощи аксикона с существенно скругленной вершиной, и результатов теоретического расчета показано, что период пульсаций интенсивности определяется формой скругления поверхности и углом при вершине конической линзы и зависит от расстояния до нее. Анализ этой зависимости позволяет восстановить форму скругления вершины конической линзы без 3D-сканирования.

Ключевые слова: бесселевы пучки, аксикон, капельные пучки.

DOI: 10.21883/PJTF.2021.20.51615.18944

В теории идеальные бесселевы пучки, формируемые при помощи аксикона, имеют свойство распространяться без расходимости и содержат бесконечную энергию [1]. На практике распределение интенсивности падающего на аксикон пучка, как правило, описывается гауссовой функцией [2], в результате чего формируется квазибесселев пучок с конечной длиной распространения, которая зависит от апертуры образующего пучка. Формируемые таким образом квазибесселевы пучки могут быть эффективно использованы для захвата и манипулирования микрообъектами [3], оптической визуализации [4], обработки материалов [5], микроскопии плоскостного освещения [6] и терагерцевой фотоники [7]. Возможности их практического использования значительно расширились после демонстрации генерации квазибесселевых пучков при помощи полупроводниковых лазеров и светодиодов $[8,9]$.

В последнее время активно изучаются квазибесселевы пучки, центральный луч которых распространяется прерывисто в виде „световых капель“ $[6,10,11]$. Такие пучки, называемые „капельными“, активно применяются, в частности, в высокоразрешающей микроскопии [11] для получения контрастного изображения толстых непрозрачных объектов без искажений, так как разрешение капельного пучка сопоставимо с разрешением стандартной оптической системы на основе гауссова пучка, а „световые капли“ сохраняют свой пространственный профиль и способны обеспечить полноценное освещение внутри полых непрозрачных объектов. При этом капельные пучки также могут быть использованы в оптогенетике для изучения динамики движения живых клеток [12] и активности нейронов [13].

Однако экспериментальные установки для формирования капельного квазибесселева пучка, использующие дорогостоящий пространственный световой модулятор $[6,10,11]$, сложны в использовании и требуют тонкой настройки. Поэтому применение аксикона со скругленной вершиной (рис. $1, a$ ) для генерация капельных квазибесселевых пучков [14-16] является привлекательной альтернативой, так как позволяет значительно упростить и удешевить экспериментальную установку.

Появление „капельной“ структуры центрального пятна квазибесселева пучка, формируемого аксиконом со скругленной вершиной, объясняется тем, что скругленная область представляет собой фокусирующую линзу, проходя через которую излучение сохраняет проекцию волнового вектора на ось симметрии, в то время как излучение, преломленное конической частью аксикона, имеет меньшую проекцию волнового вектора, величина которой определяется углом преломления [15-17]. В результате интерференции возникают осцилляции с полупериодом $\Delta z=\lambda / 2(1-\cos \gamma) \approx \lambda / \gamma^{2}$ (при $\left.\gamma \ll 1\right)$ [17], где $\lambda$ - длина волны излучения, $\gamma-$ угол, под которым свет распространяется после преломления на конической поверхности аксикона (рис. 1,a).

В настоящей работе на основе экспериментальных исследований продольного распределения интенсивности и результатов теоретического расчета показано, что полупериод пульсации интенсивности на оси не является постоянной величиной, равной $\Delta z=\lambda / \gamma^{2}$, но зависит от расстояния до скругленной вершины и определяется 

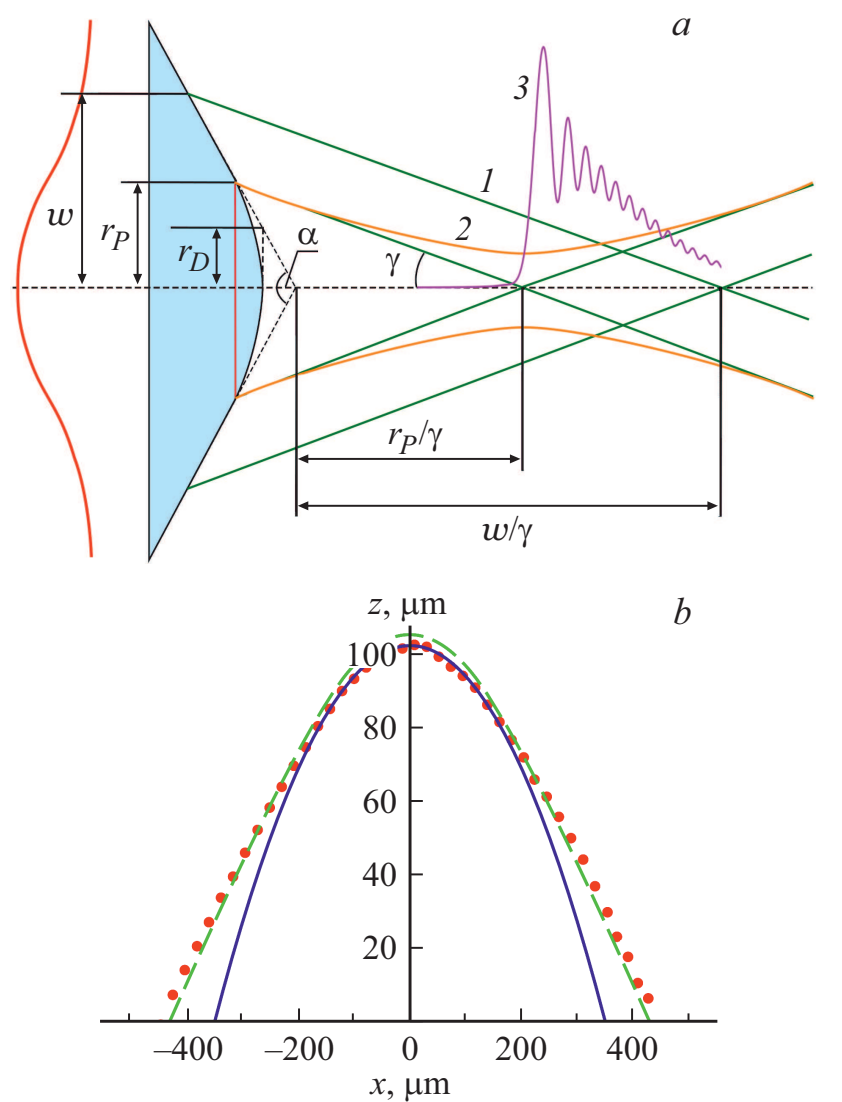

Рис. 1. $a-$ схема эксперимента: исходный гауссов пучок с радиусом $w$ падает на аксикон со скругленной вершиной и углом $\alpha=140^{\circ}\left(r_{D}\right.$ и $r_{P}$ - параметры модели, которая описывает форму поверхности аксикона, см. пояснение в тексте); излучение, прошедшее через коническую часть аксикона, распространяется под углом $\gamma$ относительно продольной координаты $z$ (линии 1 ), формируя квазибесселев пучок вблизи оси; расстояния $r_{P} / \gamma$ и $w / \gamma$ определяют начало и конец геометрической области распространения квазибесселева пучка; скругленная часть аксикона, представляющая собой линзу, фокусирует излучение в точке с координатой $z=r_{P} / \gamma$ (кривые 2); интерференция света от конической и скругленной областей формирует осцилляции продольного распределения интенсивности (кривая 3 ). $b$ - измеренный на профилометре профиль поверхности аксикона (точки), его аппроксимация параболой $v x^{2} / 2 r_{P}$ (сплошная кривая) и предложенной моделью (1) (штриховая кривая), где $v=0.364, r_{P}=220 \mu \mathrm{m}$ и $r_{H}=130 \mu \mathrm{m}$

формой поверхности аксикона. Исследование данной зависимости и использование показаний профилометра позволяют восстановить точную форму поверхности аксикона без 3D-сканирования. Исследование поверхности аксикона на профилометре позволяет получить информацию о близкой к вершине области. В свою очередь область вдали от вершины восстанавливается из результатов экспериментального исследования полупериода осцилляций.

Для исследования периода осцилляций интенсивности рассмотрим общую форму поверхности, которая задает- ся сшивкой параболы и гиперболы [18]:

$$
f(r)= \begin{cases}v\left(r_{D}+r^{2} / 2 r_{P}\right), & r \leqslant r_{0}, \\ v \sqrt{r_{H}^{2}+r^{2}}, & r>r_{0},\end{cases}
$$

где $v=\operatorname{ctg}(\alpha / 2)$ определяется углом при вершине аксикона $\alpha ; r_{P}$ и $r_{H}$ - радиусы кривизны параболической и гиперболической функций, умноженные на $v$; $r_{D}$ - расстояние от скругленной вершины до вершины идеального аксикона, деленное на $v ; r_{0}$ - точка сшивки. При этом только два параметра из четырех $\left(r_{P}, r_{H}, r_{D}\right.$, $\left.r_{0}\right)$ являются независимыми, так как на функцию (1) и на ее первую производную накладываются условия непрерывности в точке $r_{0}$. Таким образом, главным отличием рассматриваемой модели поверхности аксикона от широко известных в литературе гиперболической модели $[15,17]$ и сшивки конуса со сферой [14] или параболой [16] является наличие двух независимых параметров вместо одного. В эксперименте при помощи измерения поверхности скругленного аксикона на профилометре Sloan DECTAC 3030 и дальнейшей обработки профиля (рис. $1, b)$ нами получен первый из двух независимых параметров: $r_{P}=220 \mu \mathrm{m}$. Следует отметить, что при снятии показаний профилометра присутствует неизбежная ошибка, связанная с регистрацией сечения вне плоскости, проходящей строго через центр аксикона. Таким образом, исходя из сечения, показанного на рис. $1, b$, не представляется возможным надежно определить второй независимый параметр. В качестве второго независимого параметра, явно определяемого из эксперимента, используется расстояние $r_{H}$. Для этого нами были проведены экспериментальные исследования продольного распределения интенсивности (рис. 1,a) квазибесселева пучка, формируемого аксиконом со скругленной вершиной. Продольное распределение было получено из набора поперечных распределений интенсивности при помощи фотосьемки через равные промежутки в $10 \mu \mathrm{m}$, которые впоследствии обрабатывались с помощью программы ImageJ [19]. Угол при вершине аксикона составлял $\alpha=140^{\circ}$ (рис. 1,a), $w \approx 0.8 \mathrm{~mm}$ - радиус гауссова пучка по уровню интенсивности $1 / e$ от значения интенсивности на оси, а коэффициент преломления материала аксикона $n \approx 1.5$, что соответствует $v=\operatorname{ctg}(\alpha / 2)=0.364$ и углу преломления $\gamma=v(n-1)=0.182$. Зависимость от номера экстремума $s$ полупериода осцилляций $\Delta z(s)=z_{s+1}-z_{s}$, который определяется как расстояние между соседними экстремумами измеренного распределения интенсивности, представлена на рис. 2. При этом выражение, определяющее полупериод для рассматриваемой формы поверхности (1), имеет следующий вид [18]:

$$
\begin{aligned}
\Delta z(s) & =\frac{1}{2 \gamma}\left(\lambda / \gamma+\sqrt{r^{(0)}(s+1)^{2}-4 r_{H}^{2}}\right. \\
& \left.-\sqrt{r^{(0)}(s)^{2}-4 r_{H}^{2}}\right),
\end{aligned}
$$


где введено обозначение $r^{(0)}(s)=(s-1 / 4) \lambda / \gamma+$ $+\left(r_{H}^{2}+r_{P}^{2}\right) / r_{P}$. Выражение (2) зависит от параметров $r_{P}$ и $r_{H}$. Параметр $r_{P}$ в нашем случае известен $\left(r_{P}=220 \mu \mathrm{m}\right)$, а $r_{H}$ необходимо определить. Для угла при вершине аксикона $\alpha=140^{\circ}$ выполняется параксиальное приближение, поэтому рассматриваемый формализм является справедливым. Аппроксимируя формулой (2) полученные экспериментальные точки, определяем численное значение параметра: $r_{H}=130 \pm 14 \mu \mathrm{m}$ (рис. 2). Для наглядности на рис. 2 также построен полупериод осцилляций для двух граничных случаев: сшивки гиперболой $\left(r_{H}=220 \mu \mathrm{m}\right)$ и сшивки параболой и прямой $\left(r_{H}=0\right)$. Остальные два параметра модели (1), как упоминалось ранее, находятся из условия непрерывности функции и производной [18]: $\quad r_{D}=\left(r_{H}^{2}+r_{P}^{2}\right) / 2 r_{P}=148 \mu \mathrm{m} \quad$ и $r_{0}=\left(r_{P}^{2}-r_{H}^{2}\right)^{1 / 2}=178 \mu \mathrm{m}$. Физическая причина возникающей зависимости периода осцилляций от расстояния до вершины аксикона заключается в отклонении поверхности аксикона вдали от вершины $\left(r \gg r_{P}\right)$ от идеального конуса: $\delta f(r)=[f(r)-v r] \approx v r_{H}^{2} / 2 r$. Свет, преломленный на конической части в точке с радиусом $r$, распространяется под углом $\gamma$ к оси симметрии до точки $z=r / \gamma$, как показано на рис. $1, a$. При этом фазовый множитель $\exp \left[-i k_{0}(n-1) \delta f(r)\right]$, возникающий из-за отклонения поверхности аксикона от конической формы, также переносится из точки с радиусом $r$ в плоскости аксикона на ось симметрии $z=r / \gamma$. В результате амплитуда поля на оси приобретает дополнительный фазовый множитель, который явно зависит от расстояния $r_{H}$ :

$$
E(z) \propto \exp \left[-i k_{0}(n-1) \delta f(\gamma z)\right]=\exp \left[-i k_{0} r_{H}^{2} / 2 z\right],
$$

где $k_{0}-$ волновой вектор света в вакууме. Из формулы (3) видно, что вклад от полученного слагаемого уменьшается при увеличении продольной координаты $z$ или номера экстремума $s$. В результате полупериод стремится к постоянному значению $\lambda / \gamma^{2}$, как видно из рис. 2. Кроме того, уменьшение расстояния $r_{H}$ также приводит к подавлению фазового множителя (3), из-за чего ослабевает зависимость полупериода от продольной координаты $z$. Из рис. 2 видно, что в соответствии с данными рассуждениями аппроксимация поверхности аксикона гиперболой дает наиболее резкую зависимость периода „световых капель“ от расстояния. Переход к сшивке параболоида и конуса, напротив, приводит к обнулению фазового множителя (3) и исчезновению зависимости периода осцилляций от расстояния. Поскольку наблюдаемый эффект определяется свойствами поверхности вдали от скругленной области аксикона, полученные результаты можно с легкостью распространить на модель сшивки конуса и сферы [14].

Таким образом, экспериментальные исследования продольного распределения интенсивности квазибесселева пучка и результаты теоретического расчета показали, что период осцилляций продольного распределения интенсивности не является постоянной величиной, а зависит от расстояния до скругленной вершины. Анализ этой

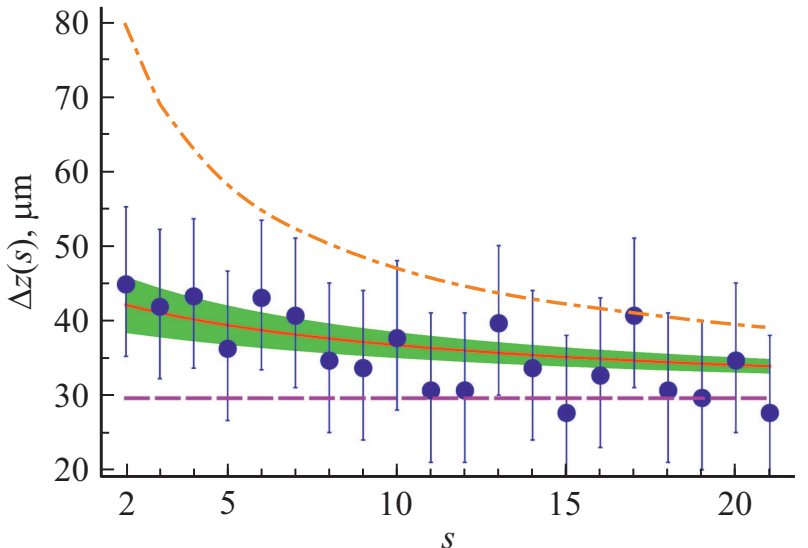

Рис. 2. Зависимость полупериода осцилляций $\Delta z$ от номера экстремума $s$, полученная из анализа экспериментальных данных (точки). Экспериментальные значения аппроксимировались формулой (2), откуда находился параметр модели $r_{H}$. Сплошная кривая показывает соответствующий полупериод для $r_{H}=130 \mu \mathrm{m}$, тонированная область - доверительный интервал для полупериода со значением доверительной вероятности 0.95. Также построены полупериоды для двух предельных случаев сшивки: штрихпунктирная кривая - сшивка гиперболической функцией $\left(r_{H}=220 \mu \mathrm{m}\right)$, штриховая кривая сшивка параболой и прямой $\left(r_{H}=0\right)$.

зависимости совместно с использованием данных линейного сканирования при помощи профилометра дает возможность восстановить форму скругления вершины конической линзы без 3D-сканирования. В перспективе полученные результаты могут быть использованы для оптимизации дизайна бутылочных пучков [20] и создания контролируемых продольных распределений интенсивности [21]. За счет варьирования периода осцилляций продольного распределения интенсивности пучка будет также меняться и длина „световых капель“, что может обеспечить новые приложения в высокоразрешающей микроскопии [11], где необходимо обеспечить подсветку полостей разного объема внутри полых непрозрачных объектов. Также данное свойство может быть эффективно использовано для лазерной резки стекла различной толщины [22].

\section{Конфликт интересов}

Авторы заявляют, что у них нет конфликта интересов.

\section{Список литературы}

[1] J. Durnin, J. Opt. Soc. Am. A, 4, 651 (1987). DOI: $10.1364 / J O S A A .4 .000651$

[2] D. McGloin, K. Dholakia, Contemp. Phys., 46, 15 (2005). DOI: $10.1080 / 0010751042000275259$

[3] B. Shao, S.C. Esener, J.M. Nascimento, E.L. Botvinick, M.W. Berns, Appl. Opt., 45, 6421 (2006). DOI: $10.1364 / A O .45 .006421$ 
[4] B. Xiong, X. Han, J. Wu, H. Xie, Q. Dai, Opt. Express, 28, 9464 (2020). DOI: 10.1364/OE.388808

[5] R. Stoian, M.K. Bhuyan, A. Rudenko, J.-P. Colombier, G. Cheng, Adv. Phys. X, 4, 1659180 (2019). DOI: $10.1080 / 23746149.2019 .1659180$

[6] G. Di Domenico, G. Ruocco, C. Colosi, E. DelRe, G. Antonacci, Sci. Rep., 8, 17178 (2018). DOI: $10.1038 / \mathrm{s} 41598-018-35006-1$

[7] Z. Wu, X. Wang, W. Sun, S. Feng, P. Han, J. Ye, Y. Zhang, Sci. Rep., 7, 13929 (2017). DOI: 10.1038/s41598-017-12524-y

[8] Г.С. Соколовский, В.В. Дюделев, С.Н. Лосев, С.А. Золотовская, А.Г. Дерягин, В.И. Кучинский, Э.У. Рафаилов, В. Сиббетт, Письма в ЖТФ, 34 (24), 75 (2008). [G.S. Sokolovskii, V.V. Dudelev, S.N. Losev, S.A. Zolotovskaya, A.G. Deryagin, V.I. Kuchnskii, E.U. Rafailov, W. Sibbett, Tech. Phys. Lett., 34 (12), 1075 (2008). DOI: 10.1134/S1063785008120262

[9] Г.С. Соколовский, В.В. Дюделев, С.Н. Лосев, А.Г. Дерягин, Д.А. Винокуров, А.В. Лютецкий, Н.А. Пихтин, С.О. Слипченко, И.С. Тарасов, С.А. Золотовская, Э.У. Рафаилов, В.И. Кучинский, В. Сиббет, Письма в ЖТФ, 36 (1), 22 (2010). [G.S. Sokolovskii, V.V. Dudelev, S.N. Losev, A.G. Deryagin, D.A. Vinokurov, A.V. Lyutetskii, N.A. Pikhtin, S.O. Slipchenko, I.S. Tarasov, S.A. Zolotovskaya, E.U. Rafailov, V.I. Kuchinskii, W. Sibbett, Tech. Phys. Lett., 36 (1), 9 (2010). DOI: $10.1134 / \mathrm{S} 1063785010010049]$.

[10] L. Li, W.M. Lee, X. Xie, W. Krolikowski, A.V. Rode, J. Zhou, Opt. Lett., 39, 2278 (2014). DOI: 10.1364/OL.39.002278

[11] G. Antonacci, G.Di Domenico, S. Silvestri, E. DelRe, G. Ruocco, Sci. Rep., 7, 17 (2017). DOI: $10.1038 / \mathrm{s} 41598-017-00042-\mathrm{w}$

[12] T.A. Planchon, L. Gao, D.E. Milkie, M.W. Davidson, J.A. Galbraith, C.G. Galbraith, E. Betzig, Nature Meth., 8, 417 (2011). DOI: 10.1038/nmeth.1586

[13] R. Prevedel, Y.-G. Yoon, M. Hoffmann, N. Pak, G. Wetzstein, S. Kato, T. Schrödel, R. Raskar, M. Zimmer, E.S. Boyden, A. Vaziri, Nature Meth., 11, 727 (2014). DOI: $10.1038 /$ nmeth. 2964

[14] Г.С. Соколовский, В.В. Дюделев, С.Н. Лосев, М. Буткус, К.К. Соболева, А.И. Соболев, А.Г. Дерягин, В.И. Кучинский, В. Сиббет, Э.У. Рафаилов, Квантовая электроника, 43 (5), 423 (2013). [G.S. Sokolovskii, V.V. Dyudelev, S.N. Losev, M. Butkus, K.K. Soboleva, A.I. Sobolev, A.G. Deryagin, V.I. Kuchinskii, V. Sibbet, E.U. Rafailov, Quant. Electron., 43 (5), 423 (2013). DOI: 10.1070/QE2013v043n05ABEH015170].

[15] Д.В. Чистяков, С.Н. Лосев, С.Х. Абдулразак, В.Ю. Мыльников, Е.А. Когновицкая, Ю.М. Задиранов, Н.Г. Дерягин, В.В. Дюделев, В.И. Кучинский, Г.С. Соколовский, Оптика и спектроскопия, 127 (5), 781 (2019). DOI: 10.21883/OS.2019.11.48515.229-19 [D.V. Chistyakov, S.N. Losev, S.H. Abdulrazak, V.Y. Myl'nikov, E.A. Kognovitskaya, Y.M. Zadiranov, N.G. Deryagin, V.V. Dudelev, V.I. Kuchinskii, G.S. Sokolovskii, Opt. Spectrosc. 127 (5), 848 (2019). DOI: $10.1134 / \mathrm{S} 0030400 X 19110079]$

[16] С.Н. Лосев, С.Х. Абдулразак, Д.В. Чистяков, В.Ю. Мыльников, Е.А. Когновицкая, И.В. Беркутов, Ю.М. Задиранов, Н.Г. Дерягин, В.В. Дюделев, В.И. Кучинский, Г.С. Соколовский, Письма в ЖТФ, 44 (19), 72 (2018). DOI: 10.21883/PJTF.2018.19.46685.17361 [S.N. Losev, S.K. Abdulrazak, D.V. Chistyakov, V.Y. Myl/nikov, E.A. Kognovitskaya, I.V. Berkutov, Y.M. Zadiranov, N.G. Deryagin, V.V. Dudelev, V.I. Kuchinskii, G.S. Sokolovskii, Tech. Phys. Lett. 44 (10), 887 (2018). DOI: $10.1134 / \mathrm{S} 1063785018100097]$.
[17] O. Brzobohatý, T. Čižmár, P. Zemánek, Opt. Express, 16, 12688 (2008). DOI: 10.1364/OE.16.012688

[18] V.Y. Mylnikov, G.S. Sokolovskii, Phys. Rev. A, in press (2021).

[19] ImageJ [Электронный ресурс]. URL: https://imagej.nih.gov/ij/

[20] T. Du, T. Wang, F. Wu, Opt. Commun., 317, 24 (2014). DOI: $10.1016 /$ j.optcom.2013.11.059

[21] N. Stsepuro, P. Nosov, M. Galkin, G. Krasin, M. Kovalev, S. Kudryashov, Appl. Sci., 10, 7911 (2020). DOI: 10.3390/app10217911

[22] J. Dudutis, P. GeČys, G. RaČiukaitis, Opt. Express, 24, 28433 (2016). DOI: $10.1364 /$ OE.24.028433 